\section{PSQ-014 NOVEL ORAL ANTICOAGULANTS VS VITAMIN K ANTAGONISTS: A COST ANALYSIS}

M Tonelli*, DA Barila', E Caiazza, M Scaldaferri, F Cattel. A.O.U. Città Della Salute e Della Scienza di Torino, Hospital Pharmacy, Turin, Italy

10.1136/ejhpharm-2019-eahpconf.447

Background In elderly patients, anticoagulants are the most commonly implicated medication in emergency department (ED) visits due to an adverse drug event (ADE): 17.6\% of all $\mathrm{ADE}$ requiring the $\mathrm{ED}$ are linked to oral anticoagulant: $50 \%$ of them require hospital admission.

Purpose The aim of the study was to assess whether the main reason for hospitalisation is related to ADE of NOACs: to evaluate the potential exposure to drug-drug interactions/assess whether contraindicated drugs have been prescribed in association with NOACs; and evaluate the economic impact associated with NOACs therapy.

Material and methods Data from 2016-2017 were retrieved from administrative and health databases: the File C2 registry which groups all patients admitted to the ED filtered using identified ICD-9-CM codes (International Classification of Diseases) related to ADE possibly induced by anticoagulants; the File $\mathrm{F}$ registry, from local health units to identify anticoagulant therapy; and the hospital discharge form (SDO) which stores clinical information about patients. File C2, File F and SDO were matched to estimate costs incurred by the healthcare system: Diagnosis Related Group (DRG) codes were analysed to evaluate the cost/patient.

Results Data of 1867 patients were extrapolated from File C2, matched with File F, through ICD9-CM related to ADE from anticoagulants: 43 patients were selected (median age $=80$ $(\sigma=12)$, male:76\%). The most frequent diagnoses were: subdural haemorrhage (31\%), iron deficiency anaemia and chronic blood loss (22\%), subarachnoid haemorrhage (9\%) due to Warfarin (75.5\%), Dabigatran (8.9\%), Rivaroxaban (8.9\%).

Crossing File C2 and SDO, 62\% of patients in treatment with anticoagulants underwent hospitalisation (average duration of 10 days) and 22/43 patients showed potential drug-drug interactions mainly due to Warfarin. The average cost per hospitalisation was significantly greater for patients treated with Warfarin versus NOACs (€900 more). The lower economic impact of cases treated with NOACs versus Warfarin per DRG (€ 56154 vs $€ 201,743)$ as for admission to the ED ( $€ 1894$ vs $€ 6,952$ ) were linked to the minor incidence of serious ADEs.

Conclusion Making a simulation, the potential saving would be proportional to the number of hospitalisations avoided, $(€ 29,106,939)$. Despite the difference in cost of the therapies shifting from AVKs to NOACs, there could be a direct economic saving related to the lower incidence of hospitalization, and indirect from the reduction of ADE.

\section{REFERENCES AND/OR ACKNOWLEDGEMENTS}

No conflict of interest.

\section{PSQ-015 DRUG INTERACTION BETWEEN NOACS AND ITRACONAZOLE: AN ITALIAN DISTRICT ANALYSIS}

E Viglione*, M Lecis, S Strobino, G Ceravolo. ASL TO3, Hospital Pharmacy, Rivoli, Italy

10.1136/ejhpharm-2019-eahpconf.448

Background New oral anticoagulant drugs (NOACs) are glycoprotein-P (gp-P) substrate, a membrane transporter protein and principally they are metabolised by CYP3A4. NOACs administration with antibiotics is not recommended because they are powerful CYP3A4 and gp-P inhibitors. It would lead to a NOACs metabolism reduction, increasing plasma concentration and, consequently, the exposure to the active substance with the risk of bleeding.

Purpose The aim of this study consisted in searching for patients with concomitant NOACs and antifungal therapies, and examining general practitioners' prescriptions.

Material and methods Prescriptions from an Italian district in 2017, in charge of the Italian national health system, were analysed. The molecules considered were NOACs: Apixaban, Edoxaban, Rivaroxaban, Dabigatran and the antifungal Itraconazole. Data have been extracted from a database S2i-italia and they have been elaborated with Access.

Results In 2017, 7404 patients were treated with NOACs and 2580 patientswith Itraconazole. Thirteen patients had concurrent prescriptions of NOACs and Itraconazole $(0.18 \%$ of all patients with NOACs prescriptions), with a median age of 72 years (range $43-83$ years). The age $\geq 75$ years' old is a risk factor because NOACs metabolism is slowed down and it is possible that it increases more plasma concentration. The NOACs molecules prescribed concurrently with Itraconazole were: Apixaban for five patients, Dabigatran for four, Rivaroxaban for three and Edoxaban for one. The average number of NOACs packs delivered to a patient was 5.5 (72 in total), the exceptions were the cases of two patients 76 years' old with 14 and 24 packs prescribed concurrently with acetylsalicylic acid for the whole analysed year, although they should have been avoided in the case of increased haemorrhagic risk.

Conclusion In 2017, 1.72\% of examined patients had NOACs and/or Itraconazole prescriptions, but only $0.18 \%$ of them had concurrent therapies, even if it was contraindicated because of the increase in bleeding risk. The advanced average age caused slowing of the metabolism: the frequent polypharmacy with the possibility of drug interaction increased the bleeding risk. It is appropriate to focus on each case and evaluate dose reduction, and make a therapeutic reconciliation, especially in elderly patients in polytherapy.

\section{REFERENCES AND/OR ACKNOWLEDGEMENTS}

'Progetto Farmamico: Interazioni TAO, AVK, NAO, Guida Medica'.

No conflict of interest.

\section{PSQ-016 UNFAVOURABLE OUTCOMES OF BLOOD TRANSFUSIONS IN HOSPITALISED ANAEMIC PATIENTS}

\begin{abstract}
${ }^{1} \mathrm{~A}$ Alcobia, ${ }^{1} \mathrm{~A}$ Soares, ${ }^{2} \mathrm{MF}$ Delerue, ${ }^{3} \mathrm{H}$ Pereira, ${ }^{4} \mathrm{H}$ Mansinho, ${ }^{5} \mathrm{~J}$ Félix, ${ }^{6} \mathrm{M}$ Afonso-Silva* ${ }^{6} \mathrm{M}$ Vargas Gomes, ${ }^{6} \mathrm{M}$ Amorim, ${ }^{6} \mathrm{M}$ Plácido, ${ }^{7} \mathrm{~S}$ Rabiais, ${ }^{8} \mathrm{~V}$ Andreozzi. ${ }^{1}$ Hospital Garcia de Orta, Pharmaceutical Services, Almada, Portugal; ${ }^{2}$ Hospital Garcia de Orta, Internal Medicine, Almada, Portugal; ${ }^{3}$ Hospital Garcia de Orta, Cardiology, Almada, Portugal; ${ }^{4}$ Hospital Garcia de Orta, Haemato-Oncology, Almada, Portugal; ${ }^{5}$ Exigo Consultores, Director, Lisbon, Portugal;; ${ }^{6}$ Exigo Consultores, Outcomes Research, Lisbon, Portugal; ${ }^{7}$ Exigo Consultores, Quantitative Department, Lisbon, Portugal; ${ }^{8}$ Exigo Consultores, Quantitative Department and Research and Innovation, Lisbon, Portugal
\end{abstract}

\subsection{6/ejhpharm-2019-eahpconf.449}

Background Guidelines recommend the administration of intravenous (IV) iron to patients with anaemia due to iron deficiency. Blood transfusions are the last resource, advised only in critical patients, as they quickly raise haemoglobin $(\mathrm{Hb})$ 
levels. However, they are also associated with deleterious outcomes.

Purpose We aimed to characterise the impact of blood transfusions in length of stay (LOS) and in-patient mortality, in a population of hospitalised anaemic patients treated with IV iron.

Material and methods This was a retrospective cohort study. Patient records from a Portuguese General Hospital, with at least one inpatient administration of iron sucrose (IS) in 2014-2015 or ferric carboxymaltose (FC) in 2016 (when FC became available), were reviewed. Adult anaemic patients with at least one $\mathrm{Hb}$ evaluation before and after the administration of IV iron were included. Endpoints assessed comprised the association of blood transfusions with LOS and in-patient mortality, adjusted for sex, age and baseline $\mathrm{Hb}$ level. Statistical analysis included a generalised linear mixed-effects model and logistic regressions, using a 5\% significance level.

Results Data was collected for 1178 patients, of which 878 were treated with IS and 300 with FC. Mean age was 63.9 and 71.1 for patients treated with IS and FC, respectively. The majority of patients were female: $61.4 \%$ and $51.3 \%$ for the groups treated with IS and FC, respectively. Average baseline $\mathrm{Hb}$ level was $8.4 \mathrm{~g} / \mathrm{dl}$ for both groups. The majority of patients required blood transfusions in both groups: $58.0 \%$ in the IS and $62.9 \%$ in the FC.

Receiving at least one blood transfusion increased the LOS by $21 \%$ (95\% CI: 8 to 35 ) in the IS group and $28 \%$ in the FC group (95\% CI: 3 to 60 ).

The in-hospital mortality risk increased 2.5-fold (95\% CI: 1.4 to 4.3 ) in patients treated with IS and who received a blood transfusion. As for patients treated with FC, in-hospital mortality was 4.3 times (95\% CI: 1.6 to 12.1 ) higher in patients who received a blood transfusion.

Conclusion Blood transfusions impacted adversely on patients' outcomes across different groups. Therefore, blood transfusions should be carefully considered, in accordance with the most recent patient blood management guidelines.

\section{REFERENCES AND/OR ACKNOWLEDGEMENTS}

Conflict of interest Corporate-sponsored research or other substantive relationships: this study was developed with financial support from Vifor Pharma. The authors had no restrictions or limitations during the study.

\section{PSQ-017 IMPLEMENTATION AND MONITORING OF A PROTOCOL FOR THE USE OF INTRAVENOUS IRON}

LS Diego, AM Tamara, CL Isabel ${ }^{*}$ CM Isabel, FG Maria Elisa. Hospital Santa Bárbara, Pharmacy, Soria, Spain

\subsection{6/ejhpharm-2019-eahpconf.450}

Background The Pharmacy and Therapeutics Committee approved in May 2017 a protocol for the prescription of intravenous iron in order to achieve the correct use of it in the hospital, and the establishment of an iron sucrose complex as first choice in admitted patients.

Purpose The objective of this study was to assess the degree of adaptation of the prescriptions to the protocol.

Material and methods We conducted a retrospective observational study from May 2017 to July 2018. In order to assess the degree of adaptation of the prescriptions it was checked if the requests were received correctly completed for type of patient (inpatient or outpatient), medical service, diagnosis and cause and patient's bodyweight, and if there were iron metabolism data (transferrin saturation, serum ferritin and iron) previous to the request.We also recorded the pharmaceutical product prescribed (ferric carboxymaltose or sucrose). Likewise, we reviewed if administered doses were correct, taking into account the theoretical deficiency calculated according to the Ganzoni formula. Dosage was considered correct if the difference between the administered dose and the theoretical deficiency did not exceed $\pm 500 \mathrm{mg}$ in ferric carboxymaltose and $\pm 200 \mathrm{mg}$ in sucrose.

Results A total of 271 prescriptions were analysed (outpatients $51.3 \%)$. The internal medicine department was the main service prescriber (47.2\%), followed by the gastroenterology department (21.8\%).

The principal medical diagnosis was anaemia. The cause was unknown in $35.5 \%$ of patients. Concerning the three main reasons for prescription, in descending order they were: need for fast iron replenishment $(56.5 \%)$, inefficiency or intestinal malabsorption syndromes $(14.7 \%)$ and intolerance to oral iron or impossibility to an oral regimen (11.4\%) The reasons were unknown in $4.1 \%$. Data of iron metabolism was not available in $34.7 \%$ of requests.

Ferric carboxymaltose was the pharmaceutical product chosen in most of the patients (54.6\%), of which $62 \%$ were outpatients. The total dose administered did not match the theoretical deficiency calculated in $41.3 \%$ of cases.

Conclusion The lack of data in many orders received in the pharmacy department makes it difficult to verify the appropriateness of the prescription to the protocol in many cases. This highlights that protocolisation is a dynamic process which requires a continuous assessment to ensure its utility.

Ferric carboxymaltose was used more frequently in iron replenishment in outpatients and iron sucrose in hospitalised patients.

\section{REFERENCES AND/OR ACKNOWLEDGEMENTS}

No conflict of interest.

\section{PSQ-018 IMPLEMENTATION OF PARENTERAL NUTRITION PRESCRIBING SOFTWARE IN A NEONATAL INTENSIVE CARE UNIT}

${ }^{1}$ A Abbassi, ${ }^{1} \mathrm{~N}$ Hasni, ${ }^{1} \mathrm{E}$ Bokri, ${ }^{2} \mathrm{M}$ Mseddi ${ }^{*},{ }^{3} \mathrm{E}$ Ben Hamida. ${ }^{1}$ Hospital Charles Nicolle, Tunis, Tunisia; ${ }^{2}$ Faculty of Pharmacy, Monastir, Tunisia; ${ }^{3}$ Neonatology, Hospital Charles Nicolle, Tunis, Tunisia

\subsection{6/ejhpharm-2019-eahpconf.451}

Background Parenteral nutrition in neonatal intensive care units is a daily activity with extreme risks. These risks are mainly related to the immaturity of neonates, a sensitive population. The computerisation of the process of prescription is a promotional tool to reduce the risks.

Purpose This study aimed to assess the interest in implementing software to help prescribers of parenteral nutrition in neonatology.

Material and methods This prospective comparative study was conducted in a neonatal unit, during a period of 3 months. It looked to evaluate the process of preparation of parenteral nutrition mixtures before and after the implementation of the prescribing software. This software was developed and validated by a team of doctors and pharmacists. The evaluation was performed by making a comparison between the errors that occurred during the manual 